\title{
NEW REPRESENTATION OF THE NON-SYMMETRIC HOMOGENEOUS BOUNDED DOMAINS IN $C_{4}^{4}$ AND $C^{3}$
}

\author{
GR. TSAGAS and G. DIMOU \\ Division of Mathematics \\ Department of Mathematics and Physics \\ Faculty of Technology \\ University of Thessaloniki, Thessaloniki, GREECE
}

(Received December 14, 1989 and in revised form March 1, 1990)

\begin{abstract}
This paper deals with the corresponding solvable Lie algebra to each of non-symmetric homogeneous bounded domains in $\mathbf{C}^{4}$ and $\mathbf{C}^{5}$ by special set of matrices. Some interesting properties of Kähler manifolds are found. The theory of $s$-structure on a complete Riemann manifold is also studied.
\end{abstract}

KEY WORDS AND PHRASES: J-Algebra, non-symmetric homogeneous bounded domains, homomorphism and Kähler manifolds.

1991 AMS SUBJECT CLASSIFICATION CODES. 53C55.

\section{INTRODUCTION.}

Let $D$ be a homogeneous bounded domain in $\mathbf{C}^{n}$. One of the problems for the homogeneous bounded domains is to classify those which are not symmetric. It is known that every homogeneous bounded domain in $\mathbf{C}^{n}, n \leq 3$, is symmetric. It has been proved that in each $\mathbf{C}^{4}$, and $\mathbf{C}^{5}$ there exists only one non-symmetric homogeneous bounded domain. In order to study these non-symmetric homogeneous bounded domains we need a method to describe these manifolds. This description permits us to understand better the geometry of these manifolds.

There were different descriptions of these non-symmetric homogeneous bounded domains. One of them is by means of Siegel domains of second kind. This description is complicated and very difficult to obtain some geometrical properties of these Kähler manifolds. Another method to describe the nonsymmetric homogeneous bounded domain is by means of $J$-algebra and normal $J$-algebra, which are special kinds of $J$-algebras. This is a general theory, which is not always clear in order to find through some geometrical properties of the non-symmetric homogeneous bounded domains which correspond to a given normal $J$-algebra.

It is known that to every non-symmetric homogeneous bounded domain corresponds a normal $J$-algebra. This normal $J$-algebra is a solvable Lie algebra.

The aim of the present paper is to describe the corresponding solvable Lie algebra to each of nonsymmetric homogeneous bounded domains in $\mathbf{C}^{4}$ and $\mathbf{C}^{5}$ by a special set of matrices. This description allows to prove some very interesting properties of these Kähler manifolds. The whole paper contains four sections. The second section contains the general theory about homogeneous bounded domains, and the relation between these manifolds and the Siegel domains of the second kind. It also gives the connection between homogeneous bounded domains and $J$-algebras and normal $J$-algebra. Finally, we study the theory of $s$-structure on a complete Riemannian manifold. 
The unique non-symmetric homogeneous bounded domain in $\mathbf{C}^{4}$ is studied in the third section. First of all we give the description of this non-symmetric homogeneous bounded domain by means of the Siegel domains of second kind. It also contains the corresponding normal $J$-algebra, which is solvable by a set of matrices. This representation of the normal $J$-algebra allows us to understand better this Kähler manifold. We give analytically the complex structure $J_{0}$ on this normal $J$-algebra $s$ and the inner product on $s$, which produces the Kähler metric on the corresponding solvable Lie group which is holomorphically isomorphic onto this non-symmetric homogeneous bounded domain in $\mathbf{C}^{4}$. We also determine the Lie homomorphism of this solvable Lie algebra and furthermore we classify which of them are isometrics. This classification permits us to prove that this non-symmetric homogeneous bounded domain admits $k$-symmetric structure.

The fourth section deals with the same problems, which are studied in the third section, for the unique non-symmetric homogeneous bounded domain in $\mathbf{C}^{5}$. The important result of this section is that this Kähler homogeneous manifold cannot carry any $s$-structure of $k$ order for any $k \in \mathbf{z}^{+}$.

\section{Homogeneous Bounded Domains}

Let $\mathbf{C}^{n}$ be the $\boldsymbol{n}$ dimensional Euclidean complex space. An open connected subset of $D$ of $\mathbf{C}^{n}$ is called domain. We denote by $G(D)$ the group of all holomorphic automorphisms of $D$. If $D$ is bounded, then it is called bounded domain in $\mathrm{C}^{n}$. Let $D$ be a bounded domain in $\mathrm{C}^{n}$. There exists on $D$ a volume element $\omega$ which is defined by

$$
\omega=(\sqrt{-1})^{n^{2}} K d z_{1} \wedge \ldots \wedge d z_{n} \wedge d \bar{z}, \wedge \ldots \wedge d \bar{z}_{n},
$$

where $z_{1}, \ldots, z_{n}$ are complex coordinates in $C^{n}$ and $K$ and Bergman function on $D$, which is positive. The Bergman function $K$ gives the Kähler metric $g$ on $D$ defined by

$$
g=\sum_{h=1}^{n} \sum_{1=1}^{n} \frac{\partial^{2} \log K}{\partial z_{h} \partial \bar{z}_{1}} d z_{h} \wedge d \bar{z}_{1}
$$

If $D$ is a bounded domain in $C^{n}$, then $(D, g)$ is a Kähler manifold and the group $G(D)$ has a structure of a Lie group. The bounded domain $D$ in $\mathrm{C}^{n}$ is called homogeneous, if the group $G(D)$ acts transitively on $D$ and therefore $D$, in this case, can be written

$$
D=G(D) / H
$$

where $H$ is the isotropy subgroup of $G(D)$ at the point $z_{0} \in C^{n}$. The relation (2.1) can also be written as follows:

$$
D=G_{0}(D) / H_{0}
$$

where $G_{0}(D)$ is the identify component of $G(D)$ and $H_{0}$ is the isotropy subgroup of $G_{0}(D)$ at $z_{0} \in D$.

Each domain $D$ in $\mathbf{C}^{n}$ is connected with a Siegel domain in the same Euclidean complex space $\mathbf{C}^{n}$. Now, we give some basic elements for a Siegel domain.

Let $V$ be a convex cone in $\mathbf{R}^{k}$. We consider the following map:

$$
F: C^{1} \times C^{1} \rightarrow C^{k} \cdot F:\left(w, w^{\prime}\right) \rightarrow F\left(w, w^{\prime}\right),
$$

having the properties.

(i) If $w$ is fixed, then the map

$$
F_{w^{\prime}}: \mathrm{C}^{1} \rightarrow \mathrm{C}^{k}, F_{w^{\prime}}: w \rightarrow F_{w^{\prime}}(w)=F\left(w, w^{\prime}\right),
$$

is complex linear. 
(ii) $\quad F\left(w^{\prime}, w\right)=\overline{F\left(w, w^{\prime}\right)}$.

(iii) $F(w, w) \in \bar{V}$, where $\bar{V}$ closure of $V, \bar{\forall} w \in \mathrm{C}^{1}$.

(iv) $F(w, w)=0 \Leftrightarrow w=0$.

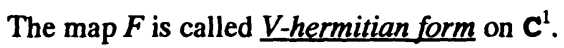

Let $V$ be a convex cone in $c^{k}$. Let $F$ be a $V$-hermitian form on $V$. We consider a subset $D(V, F)$ of $\mathrm{C}^{k} \times \mathrm{C}^{1}=\mathrm{C}^{k+1}=\mathrm{C}^{n}$, where $n=k+1$, defined as follows:

$$
D(V, F)=\left\{(z, w) \in C^{k} \times C^{1}=C^{n} / \mid m z-F(\omega, \omega) \in C^{k}\right\}
$$

which is a domain in $\mathrm{C}^{k} \times \mathrm{C}^{1}=\mathrm{C}^{n}$. This is called a Siegel domain of second kind associated to $(V, F)$. If $1=0$, then the domain $D(V, F)$ is called a Siegel domain of first kind. Hence a Siegel domain of first kind is defined by

$$
D(v)=\left\{z \in C^{k} / \mid m(z) \in V\right\},
$$

which is a special case of a Siegel domain of second kind for which the $V$-hermitian form $F$ is the zero form.

The following theorem is true ([3]).

THEOREM 2.1. Let $D^{\prime}$ be a bounded domain in $C^{n}=C^{k} \times C^{1}=C^{k+1}$. Then there is a Siegel domain of second kind $D(V, F)$ of $\mathrm{C}^{k+1}$ which is biholomorphically equivalent onto $D^{\prime}$.

If the group $G(D(V, F))$ acts on $D(V, F)$ transitively, then $D(V, F)$ is called homogeneous Siegel domain of second kind, which can be written

$$
D(V, F)=G(D(V, F)) / T,
$$

where $T$ is the isotropy subgroup of $G(D(V, F))$ at the point $\left(z_{0}, w_{0}\right)$ of $D(V, F)$.

There exists a theorem similar to Theorem 2.1.

THEOREM 2.2. Let $D^{\prime}$ be a homogeneous domain in $C^{n}=C^{k} \times C^{1}=c^{k+1}$. Then there exists a homogeneous Siegel domain of second kind $D(V, F)$ in $C^{k+1}$, which is bihomographically equivalent onto $D^{\prime}$.

To every Siegel domain of second kind $D(V, F)$ in $C^{k+1}=C^{n}$ we can associate an affine automorphism group $A F(D(V, F))$. This group $A F(D(V, F))$ plays an important role in the theory of classification of homogeneous bounded domains in $C^{n}$.

We denote by $A F\left(C^{k+1}\right)$ the group of all affine automorphisms of $C^{k+1}$. The group $A F(D(V, F))$ is defined as follows:

$$
A F(D(V, F))=\left\{\phi \in A F\left(C^{k+1}\right) / \phi(D(V, F))=D(V, F)\right\}
$$

which is a closed subgroup of $A F\left(C^{k+1}\right)$. It is known that

$$
A F\left(\mathrm{C}^{k+1}\right)=G L\left(\mathrm{C}^{k+1}\right) \cdot T\left(\mathrm{C}^{k+1}\right)
$$

where $G L\left(\mathrm{C}^{k+1}\right)$ is the group of invertible linear transformations of $\mathrm{C}^{K+1}, T\left(\mathrm{C}^{k+1}\right)$ is the group of linear transformations of $\mathbf{C}^{k+1}$ and $" \cdot "$ denotes the semi-direct product.

Let $D(V, F)$ be a Siegel domain of second kind in $\mathrm{C}^{n}=\mathrm{C}^{k+1}$. If the affine automorphism group $A F(D(V, F))$ of $D(V, F)$ acts transitively on it, then $D(V, F)$ is called homogeneous Siegel domain of second kind $D(V, F)$ in $C^{k-1}=C^{n}$ which can be written

$$
D(V, F)=A F(D(V, F) / H,
$$

where $H$ is the isotropy subgroup of $A F(D(V, F))$ at the point $(z, w) \in D(V, F)$. 
We have the following theorem.

THEOREM 2.3. Let $D^{\prime}$ be a homogeneous bounded domain in $C^{n}=C^{k+1}=C^{k} \times C^{1}$. Then there exists an affinely homogeneous Siegel domain of second kind $D(V, F)$ which is biholomorphically equivalent onto $D^{\prime}$.

From Theorems 2.2 and 2.3 we conclude that every affinely homogeneous Siegel domain of second kind can be written

$$
d(V, F)=A F(D(V, F)) / H
$$

which is also a homogeneous Siegel domain of second kind in the sense of Theorem 2.2, that means

$$
D(V, F)=G(D(V, F)) / T
$$

From (2.1) and (2.2) we obtain

$$
D(V, F)=G(D(V, F) / T=A F(D(V, F)) / H
$$

Both description (2.1) and (2.2) for a Siegel domain of second kind are used to study some properties such as space.

Let $D=G / H$ be a homogeneous bounded domain in $C^{n}=C^{k-1}$. It is known that $D$ is a complex manifold with Kähler metric $g$ defined by

$$
g=\sum_{k=1}^{n} \sum_{k=1}^{n} \frac{\partial^{2} \log f}{\partial z_{h} \partial \bar{z}_{k}} d_{z_{y}} \wedge d \bar{z}_{k}
$$

On the manifold $D$ there exists a complex structure which gives the integrable almost complex structure on it denoted by $J$.

Let $t$ and $b$ be the Lie algebras of $G$ and $H$ respectively. Let $m$ be the tangent space of $D$ at the origin 0 of $D$. Hence we obtain the decomposition:

$$
t=b+m, \quad b \cap m=0,
$$

and since $D$ is reductive we have the relation $[b, m] \subset m$. The Kähler metric $g$ on $D$ induces a Hermitian positive definite symmetric form $B$ on $m$. The almost complex structure $J$ on $D$ defines an endomorphism $J_{0}$ on $m$ with the following properties

$$
J_{0}: m \rightarrow m, \quad J_{0}: X \rightarrow J_{0}(X), J_{0}^{2}=-i d
$$

We can extend this endomorphism $J_{0}$ to another endomorphism $J_{0}{ }^{\prime}$ on $t$ which is defined as follows

$$
\begin{gathered}
J_{0}^{\prime}: t \rightarrow t, \quad J_{0}^{\prime}: X \rightarrow J_{0}^{\prime}\left(X^{\prime}\right), \quad J_{0}^{\prime 2}=-i d \\
J_{0}^{\prime}(X)=J_{0}(X), \text { if } X \in m, J_{0}^{\prime}(X)=0, \text { if } X \in b
\end{gathered}
$$

The endomorphism $J_{0}$ satisfies the following relation

$$
[X, Y]+J_{0}\left(\left[J_{0}(X), Y\right]\right)+J_{0}\left(\left[X, J_{0}(Y)\right]\right)-\left[J_{0}(X), J_{0}(Y)\right]=0, \quad X, Y \in m
$$

which is obtained from the fact that the almost complex structure on $D$ is integrable.

The relations (2.4), (2.5) and (2.6) imply that the endomorphism $J_{0}$ satisfies similar relation as (2.6), that means

$$
[X, Y]+J_{0}^{\prime}\left(\left[J_{0}^{\prime}(X), Y\right]\right)+J_{0}^{\prime}\left(\left[X, J_{0}^{\prime}(Y)\right]-\left[J_{0}^{\prime}(X), J_{0}^{\prime}(Y)\right]=0, \quad X, Y \in t\right.
$$

From the Hermitian positive definite bilinear form $B$ on $m$ we obtain a linear form $w$ on $m$ which is defined as follows

$$
w: m \rightarrow \mathbf{R}, \quad w: X \rightarrow w(X)=B(X, J(X))
$$

This linear form can be extended to another 1-form $w^{\prime}$ or $t^{\prime}$ defined by

$$
w^{\prime}: t \rightarrow \mathbf{R}, w^{\prime}: X \rightarrow w^{\prime}(X)=w\left(X_{m}\right)
$$


This linear form $w^{\prime}$ satisfies the relations

$$
\begin{gathered}
w^{\prime}\left(\left[J_{0}^{\prime}(X), J_{0}^{\prime}(Y)\right]\right)=w^{\prime}([X, Y]), \\
w^{\prime}\left(\left[J_{0}^{\prime}(X), X\right]\right)>0,
\end{gathered}
$$

for all $X, Y \in t$ and $X \notin b$.

The linear form $w$ also satisfies the relations

$$
\begin{gathered}
w\left(\left[J_{0}(X), J_{0}(Y)\right]\right)=w([X, Y]), \\
w\left(\left[J_{0}(X), X\right]\right)>0 \quad X \neq 0
\end{gathered}
$$

for all $X, Y \in m$.

Therefore from the homogeneous bounded domain $D^{\prime}=G / H$ we obtain the set $\left\{t, b, J_{0}^{\prime}, w^{\prime}\right\}$, where $J_{0}{ }^{\prime}$ is an endomorphism on $t$ having the properties (2.5) and (2.7) and $w$ a linear form on $t$ with the properties (2.10) and (2.11). The set $\left\{t^{\prime}, b, J_{0}{ }^{\prime}, w^{\prime}\right\}$ is called $J$-algebra. There exists the following theorem ([3]).

THEOREM 2.4. Let $D^{\prime}$ be a homogeneous bounded domain in $C^{n}=C^{k} \times C^{1}$. This manifold $D^{\prime}$ can be written $D^{\prime}-G / H$, where $G$ is a Lie group of analytic automorphisms of $D^{\prime}$ and $H$ the isotropy subgroup of $G$ at $z_{0} \in D^{\prime}$. We denote by $t$ and $b$ the Lie algebras of $G$ and $H$ respectively. The complex structure $J$ on $D^{\prime}$ induces a linear endomorphism $J_{0}{ }^{\prime}$ on $t$. Then there exists a linear form $w^{\prime}$ on $t$ such that the set $\left\{t, b, J_{0}^{\prime}, w^{\prime}\right\}$ be comes a $J$-algebra.

In general, there are many $J$-algebras which correspond to the same homogeneous bounded domain $D^{\prime}$, since there are a lot of Lie subgroups of the full group of analytic automorphisms on $D^{\prime}$ which act transitively on $D^{\prime}$. Let $D^{\prime}$ be a homogeneous bounded domain in $C^{n}=C^{k} \times C^{1}$. This is biholomorphically equivalent onto a homogeneous Siegel domain of second kind

$$
D(V, F)=G(D(V, F)) / T
$$

and at the same time is biholomorphically equivalent onto an affinely homogeneous Siegel domain of second kind

$$
D_{1}(V, F)=A F\left(D_{1}(V, F)\right) / H^{\prime}
$$

Let $t_{1}, b_{1}$ be the Lie algebras of $G(D(V, F))$ and $T$ respectively. There are a linear endomorphism $\left(J_{1}\right)_{0}$ and a linear form $w_{1}$ on $t_{1}$ such that the set $\left\{t_{1}, b_{1},\left(J_{1}\right)_{0}, w_{1}\right\}$ is a $J$-algebra.

Similarly, let $t_{2}, b_{2}$ be the Lie algebras of $A F\left(D_{1}(V, F)\right)$ and $H^{\prime}$ respectively. There exist a linear endomorphism $\left(J_{2}\right)_{0}$ and a linear form $w_{2}$ such that the set $\left\{t_{2}, b_{2},\left(J_{2}\right)_{0}, w_{2}\right\}$ is a $J$-algebra.

The three $J$-algebras

$$
\left\{t, b, J_{0}, w\right\}, \quad\left\{t_{1}, b_{1},\left(J_{1}\right)_{0}, w_{1}\right), \quad\left\{t_{2}, b_{2},\left(J_{2}\right)_{0}, w_{2}\right\}
$$

are isomorphic.

From the above we conclude that the study of homogeneous bounded domains in $C^{n}$ are reduced to the study of $J$-algebras and we have the theorem

THEOREM 2.5. Let $\left\{t, b, J_{0}^{\prime}, w^{\prime}\right\}$ be a $J$-algebra. Then there is always a bounded homogeneous bounded domain $D^{\prime}=G / H$ in $C^{n}=C^{k} \times C^{1}$ whose $J$-algebra is the given. There are also homogeneous Siegel domain of second kind $D(V, F)$ in $C^{n}=C^{k} \times C^{1}$ and an affinely homogeneous Siegel domain $D_{1}(V, F)$ in $C^{k} \times C^{1}$ which have a $J$-algebra the given $\left\{t, b, J_{0}^{\prime}, w^{\prime}\right\}$.

Let $D^{\prime}=G / H$ be a homogeneous bounded domain in $C^{n}=C^{k} \times C^{1}$. There exists a solvable Lie subgroup $S$ of $G\left(D^{\prime}\right)$ which can be identified with $D^{\prime}$. This domain is biholomorphically equivalent onto a homogeneous Siegel domain of second $\operatorname{kind} D(V, F)$ and simultaneously is biholomorphically equivalent 
onto an affinely homogeneous Siegel domain of second kind $D_{1}(V, F)$ in $C^{n}-C^{k} \times C^{1} . \quad D(V, F)$ and $D_{1}(V, F)$ can be written

$$
D(V, F)=G\left(D(V, F) / H^{\prime}, \quad D_{1}(V, F)=A F\left(D_{1}(V, F)\right) / T\right.
$$

It has been proved that there are solvable Lie subgroups $S^{\prime}$ and $S_{1}$ of $G(D(V, F))$ and $A F\left(D_{1}(V, F)\right)$ respectively such that

$$
D(V, F)=S^{\prime} \text { and } D_{1}(V, F)=S_{1}
$$

The corresponding $J$-algebras of $S, S^{\prime}$ and $S_{1}$ take the form

$$
\left(s, J_{0}, w\right), \quad\left(s^{\prime}, J_{0}^{\prime}, w^{\prime}\right) \text { and }\left(s_{1},\left(J_{1}\right)_{0}, w_{1}\right)
$$

respectively, where each of the Lie algebras $s, s^{\prime}$ and $s_{1}{ }^{\prime}$ are solvable.

All the properties, which are valid for the solvable $J$-algebra $(s, J, w)$ are valid for the other two $\left(s^{\prime}, J_{1}^{\prime}, w^{\prime}\right)\left(s_{1}, J_{1}, w_{1}\right)$.

Since $(s, J, w)$ is a $J$-algebra we have that $J_{0}$ is an endomorphism on $s$ with the properties.

$$
\begin{gathered}
J_{0}: s \rightarrow s, \quad J_{0}: X \rightarrow J(X), \quad J_{0}^{2}=-1, \\
{[X, Y]+J_{0}\left(\left[J_{0}(X), Y\right]\right)+J_{0}\left(\left[X, J_{0}(Y)\right]\right)-\left[J_{0}(X), J_{0}(Y)\right]=0}
\end{gathered}
$$

$w$ is a linear form on $s$ with the properties

$$
\begin{gathered}
w: s \rightarrow \mathbf{R}, w: X \rightarrow(X), \quad w\left(\left[J_{0}(X), J_{0}(Y)\right]\right)=w([X, Y]) \\
w\left(\left[J_{0}(X), X\right]\right)>0
\end{gathered}
$$

and the operator

$$
a d \tau_{0}: s \rightarrow s, a d \tau_{0}: \tau \rightarrow a d \tau_{0}(\tau)-\left[\tau_{0}, \tau\right]
$$

has only real characteristic roots, $\forall \tau_{0} \in s$, that is, $a d \tau_{0}$, as a matrix is R-triangular.

The set $(s, J, w)$ is called normal $J$-algebra. The three Lie algebras, defined by (2.16), are normal $J$-algebras, which are isomorphic.

Now, we consider a homogeneous bounded domain $D^{\prime}$ in $C^{n}$. Let $G_{1}$ be the maximal solvable Lie subgroup of $G\left(D^{\prime}\right)$ which acts transitively on $D^{\prime}$ and splits over the real numbers. We know that such a subgroup is defined uniquely up to conjugacy.

The $J$-algebras which correspond to such subgroups are normal. Let $(s, J, w)$ be a normal $J$-algebra. From this we obtain an inner product \langle\rangle on $s$ as follows

$$
\langle X, Y\rangle=w\left(\left[J_{0}(X), Y\right]\right) .
$$

We have the following theorem

THEOREM 2.6. The correspondence between normal $J$-algebras and homogeneous bounded domain in $\mathbf{C}^{n}$ is one-to-one.

Let $M$ be an $n$-dimensional connected Riemannian manifold, and $I(M)$ the group of isometrics of $M$. If there is a map $s: M \rightarrow I(M)$ such that for every $x \in M$ the image $s(x)=s_{x}$ is an isometry of $M$ having $x$ as an isolated fixed point, then the isometry $s_{x}$ is called Riemannian symmetry at $x$ or simply symmetry at $x$. The Riemannian manifold $M$ with this property is called Riemannian $s$-manifold. If there is a positive integer $k$ such that $s_{x}^{k}=i d ., \forall x \in M$, then $M$ is called a Riemannian $s$-manifold of order $k$ or simply $k$-symmetric Riemannian space. The usual Riemannian symmetric spaces are Riemannian $s$-manifolds of order 2 .

We refer three results concerning Riemannian s-manifolds [9]. 
I. The group of all isometrics $I(M)$ on a Riemannian $s$-manifold $M$ acts transitively on it.

II. Let $M$ be a Riemannian $s$-manifold. Then $M=G / H$, where $H$ is the isotropy subgroup of $G$ at any point of $M$.

III. Let $M$ be a connected Riemannian $s$-manifold. There exists another $s^{\prime}$-structure $\left\{s_{x}{ }^{\prime}: x \in M\right\}$ on $M$ such that with $\left\{s_{x}{ }^{\prime}: x \in M\right\}$ becomes a $k$-symmetric Riemannian manifold.

\section{Non-symmetric Bounded Domain in $\mathrm{C}^{4}$}

In $C^{4}$ there exists only one non-symmetric homogeneous bounded domain $D^{\prime}$. First we give the affinely homogeneous Siegel domain $D(V, F)$ which is biholomorphically equivalent onto $D^{\prime}$. We describe below $V$ and $F$.

Let $Q=H(2, \mathbf{R})$ be the vector space over $\mathbf{R}$ of all real symmetric matrices $2 \times 2$. This is a three dimensional real vector space, that is isomorphic onto $\mathbf{R}^{3}$. Therefore $k=3$ and hence $l=1$. The convex cone $V$ is $V=H^{+}(2, \mathbf{R})$, that means all positive symmetric matrices $3 \times 3$. The mapping $F$ defined by

$$
F: C \rightarrow C^{3}, \quad F:(u, v) \rightarrow F(u, v)=\left(\begin{array}{cc}
u v & 0 \\
0 & 0
\end{array}\right)
$$

Then $D\left(H^{+}(2, \mathbf{R}), F\right)$ is an affinely homogeneous Siegel domain of second kind biholomorphically equivalent onto $D^{\prime}$.

Now, our method consists of describing $D^{\prime}$ or equivalently $D\left(H^{+}(2, \mathbf{R}), F\right)$ by a normal $J$-algebra $\left(s, J_{0}, w\right)$.

We have proved that the solvable Lie algebra $s$ can be described by the set of matrices

$$
s=\left\{A=\left(\begin{array}{ccccc}
0 & x & \alpha & \gamma & \kappa \\
0 & \psi & 0 & 0 & 0 \\
0 & 0 & \beta & 0 & 0 \\
0 & 0 & 0 & \delta & 0 \\
0 & 0 & 0 & 0 & \lambda
\end{array}\right) / x, \alpha, \gamma, \kappa \in \mathbf{R} \quad \psi, \beta, \delta, \lambda \in \mathbf{R}^{*}\right\}
$$

From this construction of $s$ we conclude that the endomorphism $J_{0}$ has the form

$$
J_{0}=\left(\beta_{k l}\right), \quad \beta_{k l} \in \mathbf{R}, \quad 1 \leq k l \leq 5
$$

which must satisfy the relations (2.17) and (2.18). From these conditions and after a lot of estimates we obtain

$$
J_{0}=\left(\begin{array}{cccccccc}
\kappa & 0 & 0 & 0 & \mu & 0 & 0 & 0 \\
0 & x & 0 & 0 & 0 & \nu & 0 & 0 \\
0 & 0 & \phi & 0 & 0 & 0 & \rho & 0 \\
0 & 0 & 0 & \tau & 0 & 0 & 0 & 0 \\
-\frac{1+\kappa}{\mu} & 0 & 0 & 0 & -\kappa & 0 & 0 & 0 \\
0 & -\frac{1+x^{2}}{\nu} & 0 & 0 & 0 & -x & 0 & 0 \\
0 & 0 & -\frac{1+\phi^{2}}{\rho} & 0 & 0 & 0 & -\phi & 0 \\
0 & 0 & 0 & -\frac{1+\tau^{2}}{\sigma} & 0 & 0 & 0 & -\tau
\end{array}\right)
$$

where $\mu, v, \rho, \sigma \in \mathbf{R}^{*}$

The linear form $w$ on this Lie algebra $s$ defined by

$$
w(X)=\left\langle X_{0}, X\right\rangle
$$


where \langle\rangle the usual inner product on $s$ and $X_{0}=\left(K_{1}, K_{2}, K_{3}, K_{4}, K_{5}, K_{6}, K_{7}, K_{8}\right)$ a fixed vector. In order inat $w$ satisfies the conditions (2.19) and (2.20) we must have

$$
K_{1} \mu>0, K_{2} v>0, K_{3} \rho>0, K_{4} \sigma>0
$$

Now, we have proved the following theorem.

THEOREM 3.1. Let $D^{\prime}$ be the unique non-symmetric homogeneous bounded domain in $\mathrm{C}^{4}=\mathrm{C}^{3} \times \mathrm{C}$. The corresponding normal $J$-algebra is $\left(s, J_{0}, w\right)$, where $s, J_{0}$ and $w$ are given by (3.2), (3.3) and (3.4) respectively.

Now, we determine the solvable Lie group $S$ which corresponds to the solvable Lie algebra $s$.

We denote by $G L(S)$ the group of all nonsingular endomorphisms of $s$. The Lie algebra $g l(s)$ of $G L(S)$ consists of all endomorphisms of $s$ with the standard bracket operation $[X, Y]=X Y-Y X$. The mapping

$$
a d: s \rightarrow g l(s), \quad a d: B \rightarrow a d B
$$

where $a d B: s \rightarrow s, a d B: T \rightarrow a d B(T)=[T, B]$ is a homomorphism of $s$ onto a subalgebra $a d(s)$ of $g l(s)$. Let $\operatorname{Int}(s)$ be the analytic subgroup of $G L(s)$ whose Lie algebra is $a d(s)$. Int $(s)$ is called the adjoint group of $s$.

The group $A u t(s)$ of all automorphisms of $s$ is a closed subgroup of $G L(s)$. Thus $A u t(s)$ has a unique analytic structure under which it becomes a topological Lie subgroup of $G L(s)$. We denote by $d(s)$ the Lie algebra of $A u t(s)$. Now, the group $\operatorname{Int}(s)$ is connected so it is generated by the elements $e^{a d X}, X \in s$. Therefore Int $(s)$ is a normal subgroup of $A u t(s)$.

From the above we conclude that the solvable Lie group $S$ of $s$ is defined by

$$
S=\left\{L=\left(\begin{array}{ccccc}
1 & \frac{x}{y}\left(e^{y}-1\right) & \frac{a}{\beta}\left(e^{\beta}-1\right) & \frac{\gamma}{\delta}\left(e^{5}-1\right) & \frac{\kappa}{\lambda}\left(e^{\lambda}-1\right) \\
0 & e^{y} & 0 & 0 & 0 \\
0 & 0 & e^{\beta} & 0 & 0 \\
0 & 0 & 0 & e^{\delta} & 0 \\
0 & 0 & 0 & 0 & e^{\lambda}
\end{array}\right) / \begin{array}{c}
x, a, \gamma, k \in \mathbf{R} \\
y, \beta, \delta, \lambda \in \mathbf{R}^{*}
\end{array}\right\}
$$

The inner product on the solvable Lie algebra $s$ is defined by

$$
\langle X, Y\rangle=w\left(\left[J_{0} X, Y\right]\right)
$$

where $w$ is given by (3.4). This inner product determines the Kähler metric on $S$ which is essentially the Bergmann metric on it.

Now we can state the following theorem.

THEOREM 3.2. The homogeneous non-symmetric bounded domain in $\mathbf{C}^{4}$ is biholomorphically isomorphic onto the solvable Lie group $S$ defined by (3.7). The Kähler metric $g$ on $S$ is defined by the relation (3.8).

Let $F$ be a Lie automorphism on $s$. This $F$ can be represented by the matrix

$$
F=\left(\begin{array}{cccccccc}
a_{11} & 0 & 0 & 0 & a_{15} & 0 & 0 & 0 \\
0 & a_{22} & 0 & 0 & 0 & a_{26} & 0 & 0 \\
0 & 0 & a_{33} & 0 & 0 & 0 & a_{37} & 0 \\
0 & 0 & 0 & a_{44} & 0 & 0 & 0 & a_{48} \\
0 & 0 & 0 & 0 & 1 & 0 & 0 & 0 \\
0 & 0 & 0 & 0 & 0 & 1 & 0 & 0 \\
0 & 0 & 0 & 0 & 0 & 0 & 1 & 0 \\
0 & 0 & 0 & 0 & 0 & 0 & 0 & 1
\end{array}\right)
$$


which becomes an isometry with respect to the inner product

$$
\langle X, Y\rangle=\left\langle X_{0},[J X, Y]\right\rangle=w([X, Y])
$$

when

$$
\begin{array}{ccc}
a_{11}= \pm 1, & a_{22}= \pm 1, \quad a_{33}= \pm 1, \quad a_{44}= \pm 1, & a_{15}=\frac{\kappa \mu}{1+k^{2}}\left(\frac{1-a_{11}}{a_{11}}\right) \\
a_{26}=\frac{v x}{1+x^{2}}\left(\frac{1-a_{22}}{a_{22}}\right), & a_{37}=\frac{\phi \rho}{1+\phi^{2}}\left(\frac{1-a_{33}}{a_{33}}\right), \quad a_{48}=\frac{\sigma \tau}{1+\tau^{2}}\left(\frac{1-a_{44}}{a_{44}}\right),
\end{array}
$$

Therefore if $F$ is an isometry, then it has the form

$$
F_{\text {isom }}=\left(\begin{array}{cccccccc}
a_{11} & 0 & 0 & 0 & \frac{x \psi\left(1-a_{11}\right)}{\left(1+\kappa^{2}\right) a_{11}} & 0 & 0 & 0 \\
0 & a_{22} & 0 & 0 & 0 & \frac{v\left(1-a_{22}\right)}{\left(1+x^{2}\right) a_{22}} & 0 & 0 \\
0 & 0 & a_{33} & 0 & 0 & 0 & \frac{\psi p}{1+\psi^{2}} \frac{1-a_{33}}{a_{33}} & 0 \\
0 & 0 & 0 & a_{44} & 0 & 0 & 0 & \frac{\sigma r}{1+\tau^{2}} \frac{1-a_{44}}{a_{44}} \\
0 & 0 & 0 & 0 & 1 & 0 & 0 & 0 \\
0 & 0 & 0 & 0 & 0 & 1 & 0 & 0 \\
0 & 0 & 0 & 0 & 0 & 0 & 1 & 0 \\
0 & 0 & 0 & 0 & 0 & 0 & 0 & 1
\end{array}\right)
$$

where $a_{11}= \pm 1, a_{22}= \pm 1, a_{33} \pm 1, a_{44} \pm 1$.

From the form $F_{\text {isom }}$ we obtain that it has the eigenvalue 1 with multiplicity at least 4 times. Therefore we have proved the following theorem.

THEOREM 3.3. The homogeneous non-symmetric bounded domain in $\mathrm{C}^{4}$ with the standard Kähler metric does not admit any $k$-symmetric structure.

\section{Non-symmetric Bounded Domain in $\mathrm{C}^{4}$}

In $\mathrm{C}^{5}$ there is only one non-symmetric homogeneous bounded domain $D^{\prime}$. It is well known that there exists the affinely homogeneous Siegel domain $D(V, F)$ which is biholomorphically equivalent onto $D^{\prime}$. Therefore we need $V$ and $F$ which are described below.

The convex cone $V$, in this case, is the same as in paragraph 3, that is $V=H^{+}(2, \mathbf{R})$. The mapping $F$ is defined by

$$
F: \mathbf{C}^{2} \times \mathbf{C}^{2} \rightarrow H(2, \mathbf{R}), F:\left(u=\left(\begin{array}{l}
u_{1} \\
u_{2}
\end{array}\right), v=\left(\begin{array}{l}
v_{1} \\
v_{2}
\end{array}\right)\right) \rightarrow F(u, v)=\frac{1}{2}(u t \bar{v}+\bar{v} u)=\left(\begin{array}{cc}
u_{1} \bar{v}_{1} & \frac{u_{1} \bar{v}_{2}+u_{2} \bar{\nu}_{1}}{2} \\
\frac{u_{1} \bar{v}_{2}+u_{2} \bar{v}_{1}}{2} & u_{2} \bar{v}_{2}
\end{array}\right)
$$

Hence we have, in this case, $k=3$ and $1=2$. From above we conclude that $D\left(H^{+}(2, \mathbf{R}), F\right)$ is an affinely homogeneous Siegel domain of second kind biholomorphically onto $D^{\prime}$.

Now, our method consists of describing $D^{\prime}$ or equivalently $D\left(H^{+}(2, \mathbf{R}), F\right)$ by a normal $J$-algebra $\left(s, J_{0}, w\right)$.

We have proved that the solvable Lie algebra $s$ can be described by the set of matrices. 


$$
s=\left\{A=\left(\begin{array}{ccccc}
1 & \chi_{1} & \chi_{2} & \chi_{3} & \chi_{4} \\
0 & \chi_{1} & 0 & 0 & 0 \\
0 & 0 & \chi_{2} & 0 & 0 \\
0 & 0 & 0 & \chi_{3} & 0 \\
0 & 0 & 0 & 0 & \chi_{4}
\end{array}\right) / \begin{array}{c}
\chi_{1}, \chi_{2}, \chi_{3} \chi_{4} \in \mathbf{R} \\
\psi_{1}, \psi_{2}, \psi_{3}, \psi_{4} \in \mathbf{R}^{\cdot}
\end{array}\right\}
$$

From this construction of $s$ we conclude that the endomorphism $J_{0}$ has the form

$$
J_{0}=\left(\beta_{k l}\right), \quad \beta_{k l} \in \mathbf{R} \quad 1 \leq k, 1 \leq 5
$$

which must satisfy the relations (2.17) and (2.18).

From these conditions and after a lot of calculations we have

$$
J_{0}=\left(\begin{array}{cccccccccc}
\rho_{1} & 0 & 0 & 0 & 0 & q_{1} & 0 & 0 & 0 & 0 \\
0 & \rho_{2} & 0 & 0 & 0 & 0 & q_{2} & 0 & 0 & 0 \\
0 & 0 & \rho_{3} & 0 & 0 & 0 & 0 & q_{3} & 0 & 0 \\
0 & 0 & 0 & \rho_{4} & 0 & 0 & 0 & 0 & q_{4} & 0 \\
0 & 0 & 0 & 0 & \rho_{5} & 0 & 0 & 0 & 0 & q_{5} \\
-\frac{1+\rho^{2}}{q_{1}} & 0 & 0 & 0 & 0 & \rho_{1} & 0 & 0 & 0 & 0 \\
0 & -\frac{1+\rho_{2}^{2}}{q_{2}} & 0 & 0 & 0 & 0 & \rho_{2} & 0 & 0 & 0 \\
0 & 0 & -\frac{1+\rho_{3}^{2}}{q_{3}} & 0 & 0 & 0 & 0 & \rho_{3} & 0 & 0 \\
0 & 0 & 0 & -\frac{1+\rho_{4}^{2}}{q_{4}} & 0 & 0 & 0 & 0 & \rho_{4} & 0 \\
0 & 0 & 0 & 0 & -\frac{1+\rho_{5}^{2}}{q_{5}} & 0 & 0 & 0 & 0 & \rho_{5}
\end{array}\right)
$$

where $\rho_{i} \in \mathbf{R}, i=1, \ldots, 5, q_{i} \in \mathbf{R}^{*} i=1, \ldots, 5$.

The linear form $w$ on this Lie algebra $s$ is defined as follows

$$
w(X)=\left\langle X_{0}, X\right\rangle
$$

where \langle\rangle is the usual inner product on $s$ and $X_{0}\left(\kappa_{1}, \kappa_{2}, \kappa_{3}, \kappa_{4}, \kappa_{5}, \kappa_{6}, \kappa_{7}, \kappa_{8}, \kappa_{9}, \kappa_{10}\right)$ a fixed vector. In order $w$ to satisfy the conditions (2.19) and (2.20) we must have

$$
q_{1} \kappa_{1}>0, q_{2} \kappa_{3}>0, q_{4} \kappa_{4}>0, q_{5} \kappa_{5}>0
$$

Now, we have proved the following theorem

THEOREM 4.1. Let $D$ be the unique non-symmetric homogeneous bounded domain in $C^{5}=C^{3} \times C^{2}$. The corresponding normal $J$-algebra is $\left(s, J_{0}, w\right)$, where $s, J_{0}$ and $w$ are given by (4.2) and (4.3) respectively.

Now, we determine the solvable Lie group $S$ which corresponds to the solvable Lie algebra $s$. We denote by $G L(s)$ the group of nonsingular endomorphisms of $s$. The Lie algebra $g l(s)$ of $G L(s)$ consists of all endomorphisms of $s$ with the standard bracket operations.

$$
[X, Y]=X Y-Y X \text {. }
$$

The mapping

$$
\text { ad }: s \rightarrow g l(s), \text { ad }: B \rightarrow a d B
$$

where

$$
a d B: s \rightarrow s, a d B: T \rightarrow a d B(T)=[T, B]
$$

is a homomorphism of $s$ onto a subalgebra $a d(s)$ of $g l(s)$. Let int $(s)$ be the analytic subgroup of $G L(s)$ whose Lie algebra is $a d(s)$. It is known that $\operatorname{Int}(s)$ is called the adjoint group of $s$. 
The group $A u t(s)$ of all automorphisms of $s$ is a closed subgroup of $G L(s)$. Thus $A u t(s)$ has a unique analytic structure under which it becomes a topological subgroup of $G L(s)$. We denote by $d(s)$ the Lie algebra of $a u t(s)$. Now, the group $\operatorname{Int}(s)$ is connected, so it is generated by the elements $e^{a d(x)}, X \in s$. Therefore $\operatorname{Int}(s)$ is a normal subgroup of $\operatorname{Aut}(s)$.

From the above we conclude that the solvable Lie group $S$ of $s$ is defined by

$$
S=\left\{L=\left(\begin{array}{cccccc}
1 & \frac{x_{1}}{\psi_{1}}\left(e^{\psi_{1}}-1\right) & \frac{x_{2}}{\psi_{2}}\left(e^{\psi_{2}}-1\right) & \frac{x_{3}}{\psi_{3}}\left(e^{\psi_{3}}-1\right) & \frac{x_{4}}{\psi_{4}}\left(e^{\psi_{4}}-1\right) & \frac{x_{s}}{\psi_{5}}\left(e^{\psi_{s}}-1\right) \\
0 & e^{\psi_{1}} & 0 & 0 & 0 & 0 \\
0 & 0 & e^{\psi_{2}} & 0 & 0 & 0 \\
0 & 0 & 0 & e^{\psi_{3}} & 0 & 0 \\
0 & 0 & 0 & 0 & e^{\psi_{4}} & 0 \\
0 & 0 & 0 & 0 & 0 & e^{\psi_{s}}
\end{array}\right) / \begin{array}{l}
x_{j} \in \mathbf{R} i=1, \ldots, 5 \\
\psi_{i} \in \mathbf{R}^{*} i=1, \ldots, 5
\end{array}\right\}
$$

The inner product on the solvable Lie algebra $s$ is given by (4.3). This inner product determines the Kähler on $S$ which is essentially metric the Bergman metric on it. Now, we can state the following theorem.

THEOREM 4.2. The homogeneous non-symmetric bounded domain in $\mathbf{C}^{5}$ is biholomorphically isomorphic onto the solvable Lie group $S$ defined by (4.5). The Kähler metric $g$ on $S$ defined by the relation (4.3).

Let $F$ be a Lie automorphism on $s$. This $F$ can be represented by the matrix

$$
F=\left(\begin{array}{cccccccccc}
a_{11} & 0 & 0 & 0 & 0 & a_{16} & 0 & 0 & 0 & 0 \\
0 & a_{22} & 0 & 0 & 0 & 0 & a_{27} & 0 & 0 & 0 \\
0 & 0 & a_{33} & 0 & 0 & 0 & 0 & a_{38} & 0 & 0 \\
0 & 0 & 0 & a_{44} & 0 & 0 & 0 & 0 & a_{49} & 0 \\
0 & 0 & 0 & 0 & a_{55} & 0 & 0 & 0 & 0 & a_{51} \\
0 & 0 & 0 & 0 & 0 & 0 & 1 & 0 & 0 & 0 \\
0 & 0 & 0 & 0 & 0 & 0 & 0 & 1 & 0 & 0 \\
0 & 0 & 0 & 0 & 0 & 0 & 0 & 0 & 1 & 0 \\
0 & 0 & 0 & 0 & 0 & 0 & 0 & 0 & 0 & 1
\end{array}\right)
$$

which becomes an isometry with respect to the inner product

$$
\langle X, Y\rangle=\left\langle X,\left[J_{0} X, Y\right]-w([X, Y])\right.
$$

when

$$
\begin{gathered}
a_{11}= \pm 1, a_{22}= \pm 1, a_{33}= \pm 1, a_{44}= \pm 1, a_{55}= \pm 1 \\
a_{16}=\frac{p_{1} q_{1}}{1+p_{1}^{2}} \frac{1-a_{11}}{a_{11}}, a_{27}=\frac{p_{2} q_{2}}{1+p_{2}^{2}} \frac{1-a_{22}}{a_{22}}, a_{38}=\frac{p_{3} p_{3}}{a_{33}} \frac{1-a_{33}}{a_{33}} \\
a_{49}=\frac{p_{4} p_{4}}{1+p_{4}^{2}} \frac{1-a_{44}}{a_{44}}, a_{51}=\frac{p_{5} p_{5}}{1+p_{5}^{2}} \frac{1-a_{55}}{a_{55}}
\end{gathered}
$$

If $F$ is an isometry, then it has the form 


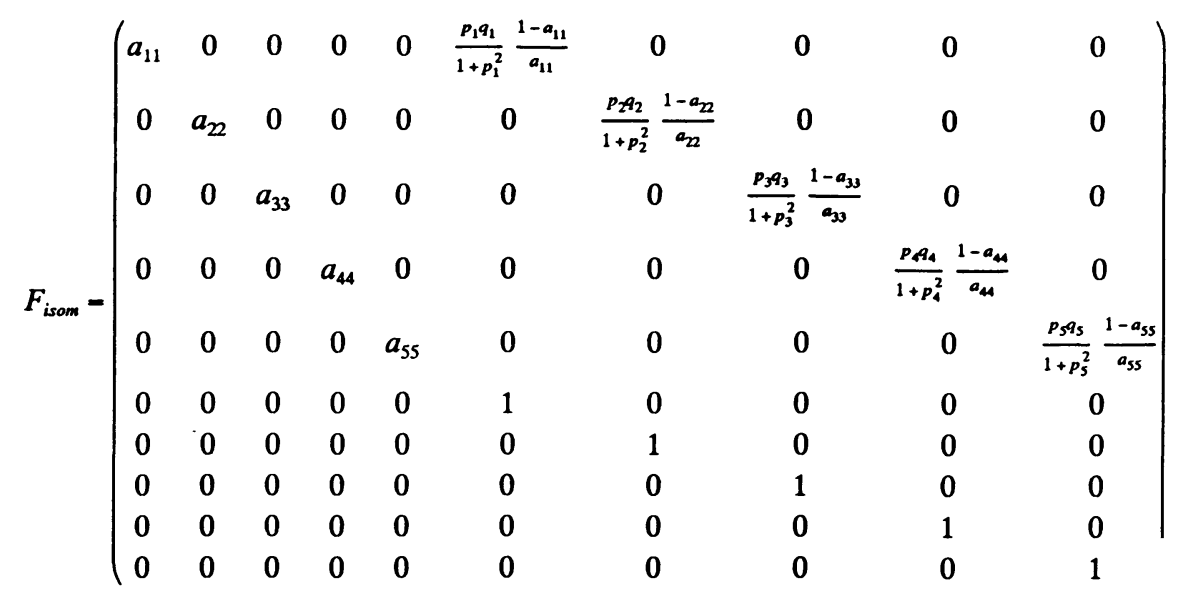

where $a_{11}= \pm 1, a_{22}= \pm 1, a_{33}= \pm 1, a_{44}= \pm 1, a_{55}= \pm 1$.

From the form of $F_{\text {isom }}$ we obtain that it has the eigenvalue 1 with multiplicity at least 4 . Therefore we have proved the following theorem.

THEOREM 4.3. The homogeneous non-symmetric bounded domain in $C^{5}$ with the standard Kähler metric does not admit any $k$-symmetric structure.

\section{REFERENCES}

1. CARTAN, E. Sur les domains bornes homogeneous de l'espace de $\mathbf{n}$ variables complexes, $\mathrm{Abh}$. Math. Sem., Hamburg Univ. 11 (1936), 116-162.

2. HANO, J. On Kählerian homogeneous of unimodular, Am. J. Math. 78 (1957), 885-900.

3. KANEYUKI, S. Homogeneous Bounded Domains and Sieger Domains, Springer-Verlag, New York (1971).

4. KOSZUL, J. Sur la forme hermitienne canonique des espaces homogenes complexes, Can. J. Math. Z (1955), 562-576.

5. LEDGER, A. and OBATA, M. Affine and Riemannian s-manifolds, J. Differ. 2 (1968), 451-459.

6. MOTSUSHIMA, Y. Sur les espaces homogenes Kähleriens d un groupe de Lie reducti, Nagoya Math. J. 11 (1957), 56-60.

7. PYATETSKIJ-SHAPIRO, J. On a problem proposed by E. Cartan, Dokl. Akad. Nauk, SSSR, 124 (1959), 272-273.

8. PYATETSKIJ-SHAPIRO, J. Automorphic Function and the Geometry of Classical Domains Gordon \& Breach, New York (1969).

9. TSAGA, GR and LEDGER, A. Riemannian s-manifolds, J. Diff. Geom., Vol. 12, No. 3 (1977), pp. 333-343. 


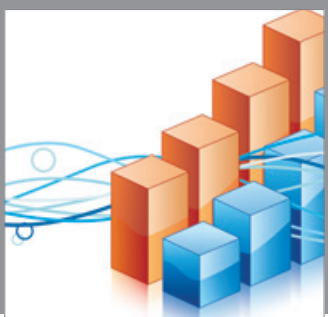

Advances in

Operations Research

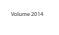

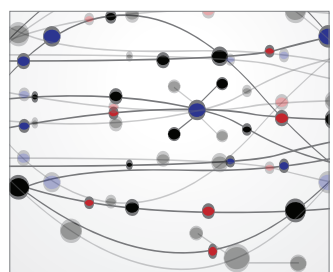

\section{The Scientific} World Journal
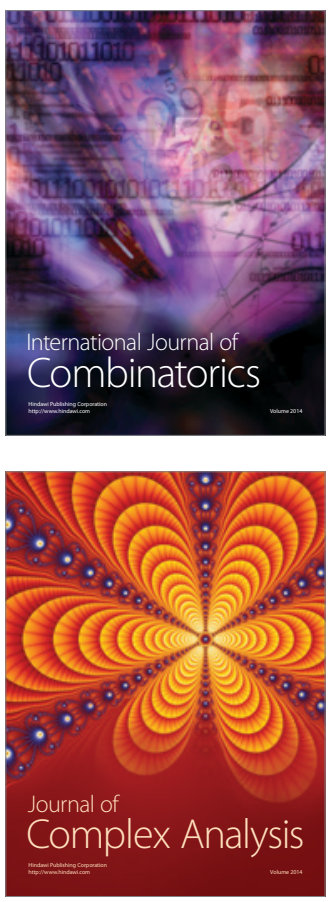

International Journal of

Mathematics and

Mathematical

Sciences
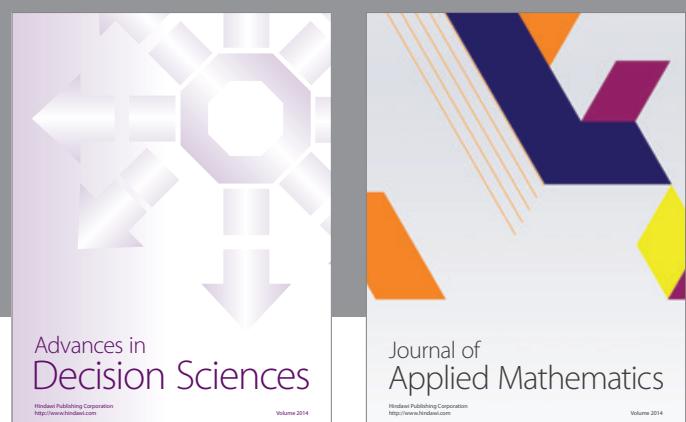

Journal of

Applied Mathematics
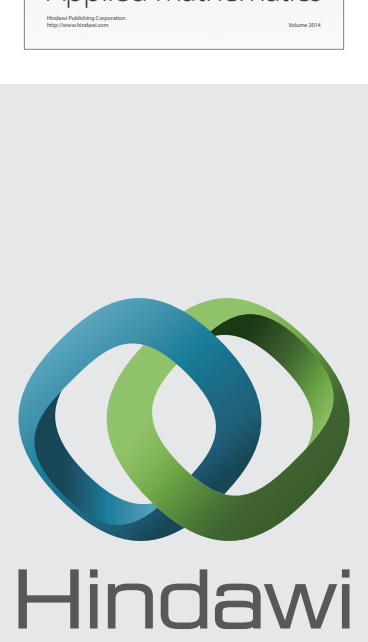

Submit your manuscripts at http://www.hindawi.com
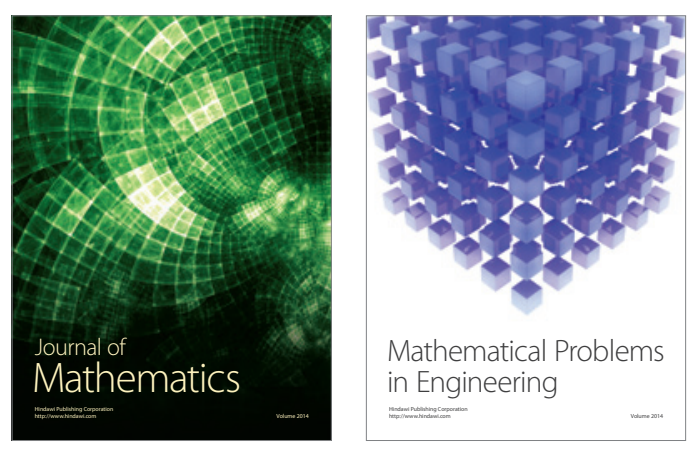

Mathematical Problems in Engineering
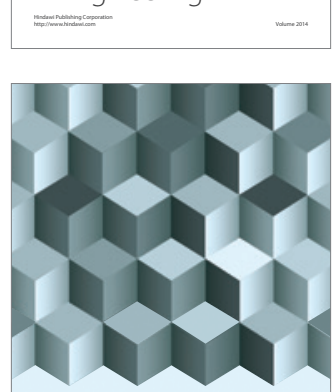

Journal of

Function Spaces
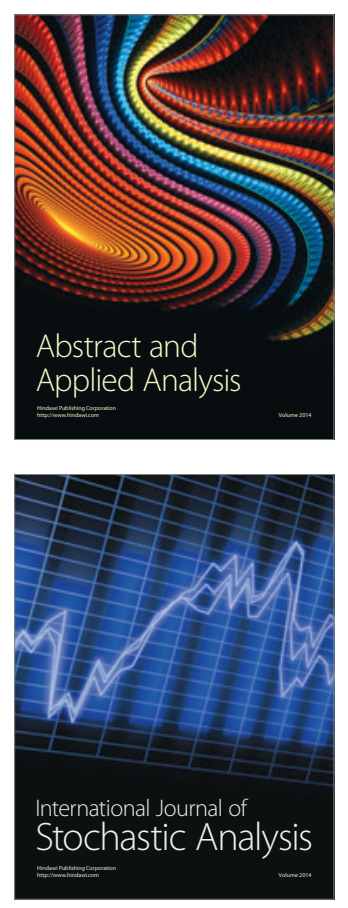

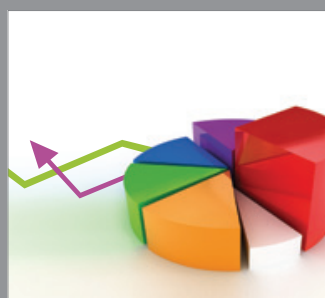

ournal of

Probability and Statistics

Promensencen
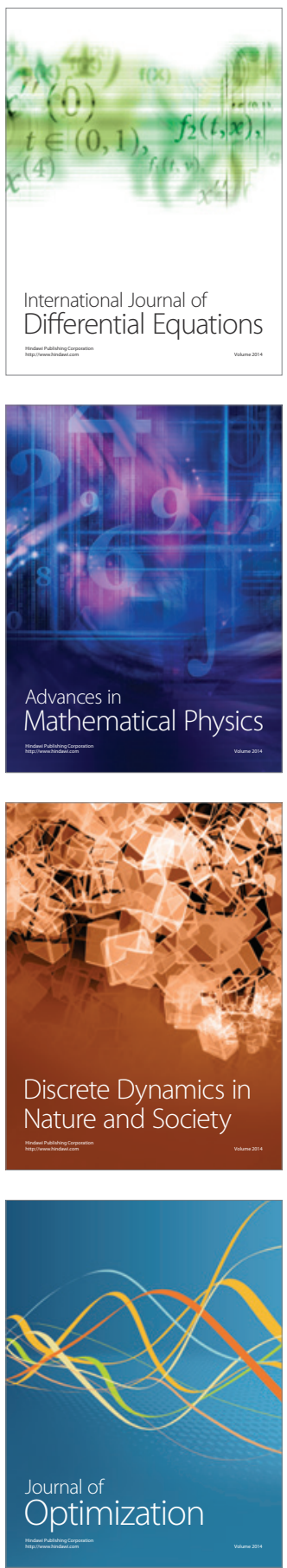